\title{
The Mental Health of Adults with Developmental Disabilities in Ontario: Lessons from Administrative Health Data
}

Yona Lunsky, Robert Balogh, Anna Durbin, Avra Selick, Tiziana Volpe and Elizabeth Lin

\begin{abstract}
Adults with developmental disabilities have increased rates of mental illness and addiction, in addition to being more likely to experience physical health issues. This can lead to high rates of hospital and community-based healthcare. Population-based administrative health data can help in identifying the extent of problems experienced and target areas for policy and practice changes.
\end{abstract}

\section{The Issue}

Mental healthcare costs total over $\$ 50$ billion a year in Canada, affecting more than 6.7 million people (Mental Health Commission of Canada 2013; Ratnasingham et al. 2012). Adults with developmental disabilities (DDs) such as Down syndrome or autism have a high rate of mental health issues and face significant barriers when accessing appropriate and timely mental healthcare (Lunsky et al. 2007, Lunsky et al. 2013c). Such challenges can lead to additional problems for them and their families.

There is currently no national strategy or approach for addressing the mental health needs of this population, and the training of mental healthcare professionals in the field of DDs is extremely limited. The situation is further complicated by the fact that the required services and supports for this group are spread across the health and social service sectors. Just how big an issue this is in our country is difficult to know with limited data. Up until recently, identifying persons with DDs in existing databases was not routinely done, leading to a lack of data and knowledge regarding their mental health diagnoses and mental health service use.

In Ontario, we had a unique opportunity to explore mental health concerns and service utilization patterns by linking data from social services to data from health services at the Institute for Clinical Evaluative Sciences (Lin et al. 2014). This initiative, which began in 2010, is part of the Health Care Access Research and Developmental Disabilities Program (H-CARDD; https://www.porticonetwork.ca/web/hcardd), a partnership between scientists, clinicians, policy makers, patients and families. We summarize here findings from the program thus far that have important implications for mental health policy and service delivery.

\section{Key Findings Greater prevalence of mental illness and addiction}

Psychiatric disorders were first described in a cohort of 43,549 adults with DDs that were identified from administrative health data (Lunsky et al. 2012) and then further explored with a larger linked cohort in our Atlas on the Primary Care of Adults with Developmental Disabilities in Ontario (Lunsky et al. 2013b). The Atlas examined a cohort of 66,484 adults with DDs in 2009-2010 that had been identified by linking data to diagnostic information from health and social services. This cross-sector linkage was important because about one-third of individuals could only be identified as having DDs through one of the two data sources. We found that psychiatric disorder diagnoses were more common in adults with DDs compared to those without DDs. Our most recent findings (Lin et al. $2016 \mathrm{~b}$ ) showed that in a two-year period, $44 \%$ of adults with DDs had a mental illness diagnosis and 6\% had an addiction diagnosis (Lin et al. 2016a). Chronic diseases such as diabetes, hypertension, chronic obstructive pulmonary disease and asthma were more common among adults with DDs and a mental illness or addiction compared to those without these diagnoses (Lin et al. 2016b).

Differential prevalence rates of mental illness are apparent by young adulthood, and specific subgroups within the DD population may be at greater risk for mental illness than others are. In a study of 18-24-year-old adults, those with autism had even higher rates of mental illness diagnoses (52\%) than their counterparts with other DDs (39\%; Weiss et al. 2018).

Higher rates of mental illness and addiction have also been found in diverse subpopulations, with implications for both their mental and physical healthcare. For example, in an H-CARDD study on pregnancies, $51 \%$ of women with DDs were found to have a mental illness or addiction diagnosis in 
the two years prior to conception compared to $28 \%$ of those without DDs (Brown et al. 2016). It is not surprising therefore that those with DDs experienced more psychiatric complications in the postpartum period, as indicated by a high number of emergency department (ED) visits and hospitalizations for mental health reasons (Brown et al. 2017). H-CARDD research on Ontario adults with HIV and DDs revealed higher rates of addictions (24\%) and psychotic disorders $(21 \%)$ compared to other adults with HIV only ( $10 \%$ and $2 \%$, respectively; Lunsky et al. 2017), which adds to the complexity of treatment.

\section{Impact of Comorbid Psychiatric Disorders on Health Service Use and Prescribing Patterns}

Our research has shown that adults with DDs, mental illness and addiction are more likely to use health services and use them repeatedly (Lin et al. 2016b). Primary care use, psychiatry use and ED use over a two-year period are more likely in adults with DDs and mental illness compared to adults with mental illness only, with frequent ED visits (five or more in two years) also being more common (Lunsky et al. 2012). Follow-up psychiatric care can be a problem; $42 \%$ of adults with DDs who had a psychiatric ED visit did not receive any outpatient follow-up in the month after their visit (Balogh et al. 2013), despite guidelines emphasizing its importance (Sullivan et al. 2011). Not only are hospitalizations and ED visits more common among those with DDs and a mental illness or addiction, but repeat admissions within 30 days of discharge are 1.7 times more likely compared to those with a mental illness or addiction only (Balogh et al. 2018). The issue of alternate level of care days in hospital when there is a psychiatric disorder combined with a DD has also been flagged as a concern (Lin et al. 2016b). Together, these findings suggest that interventions are needed to better meet the needs of this group, both within and outside of hospital.

Because the majority of adults with DDs receive income benefits through the Ontario Disability Support Plan (ODSP), patterns of medication use can be studied at the population level through the Ontario Drug Benefit claims database. The 2013 Atlas reported that adults with DDs and a comorbid psychiatric diagnosis were more likely to be dispensed five or more medications concurrently (29.4\%) than adults with DDs and no mental illness diagnosis (13.4\%). The three most commonly prescribed medication classes filled by ODSP recipients were psychotropic in nature, with antipsychotics being the most common (Cobigo et al. 2013). Follow-up research showed that over a six-year period, $39.2 \%$ of 51,881 adults with DDs filled at least one antipsychotic prescription (Lunsky et al. 2017). These rates were even higher in a subgroup of adults living in group home settings (56\%). Forty per cent of adults with a filled antipsychotic prescription had a serious mental illness diagnosis, but $29 \%$ had no psychiatric diagnosis. The high rates of antipsychotic medications dispensed to this population are a major concern in Ontario and elsewhere. Future research can explore predictors of new prescriptions and the quality of medication monitoring more closely for antipsychotics as well as other psychotropic medications.

\section{Implications}

Data on mental illness and addiction diagnoses and service use of adults with DDs are essential to the development of provincial and national policies and best practices. Unfortunately, without registries of people with DDs, it is difficult to track this information. In the absence of a registry, linked administrative data from across sectors make it possible to measure health and health service outcomes at the population level. This is particularly important for DD, because identification of these disabilities occurs across sectors and not only in healthcare. Currently, Manitoba has the greatest capacity to comprehensively link data between sectors, including health, social services, children's services, justice and education (www.umanitoba.ca/centres/mchp). Such linked data can be a rich source of information that can be used to inform and develop policy and strategy and identify who should be targeted for interventions. Over time, such data can not only identify problems but also indicate whether efforts to improve mental health supports are working.

There is a need for nation-wide information on this population, similar to the work on mental health and addiction quality indicators completed with five provinces in 2017 for the general population (Butler et al. 2017). Some provinces already have the capacity to do this for persons with DDs. Some work can also be done at a national level using information from the Canadian Institute for Health Information, but because this includes only people who were hospitalized, a number of people with DDs would be excluded from the results if this approach was taken.

Higher rates of mental illness and addictions combined with higher rates of hospital-based healthcare among Ontario adults with DDs point to the need for policies and practices that better support this group. Decisions concerning how to decrease barriers to mental healthcare and improve mental health for adults with DDs in Ontario and other parts of Canada should be sure to consider the perspectives of service users and service providers, as well as policy makers across sectors. An emphasis on proactive patient- and family-centred care is key. Linkages between hospital- and community-based care are required, particularly to assist with transitions to and from hospital. Given the high rates of medical comorbidities in this population, models of care that consider the overlap between mental health and physical health and between psychiatry and family medicine are required (Lunsky et al. 2013a). HQ 


\section{References}

Balogh, R., E. Lin, K. Dobranowski, A. Selick, A.S. Wilton and Y. Lunsky. 2018. "All Cause, 30-Day Readmission in Persons with Intellectual and Developmental Disabilities and Mental Illness." Psychiatric Services 69(3): 353-57.

Balogh, R., J.R. Wood, Y. Lunsky, H. Ouellette-Kuntz, A.S. Wilton, V. Cobigo et al. 2013. "Chronic Disease Management." In Y. Lunsky, J.E. Klein-Geltink and E.A. Yates, eds. Atlas on the Primary Care of Adults with Developmental Disabilities in Ontario. Toronto, ON: Institute for Clinical Evaluative Sciences and Centre for Addiction and Mental Health. Retrieved February 23, 2018. <http://www. ices.on.ca/ -/media/Files/Reports/2013/Atlas-on-developmentaldisabilities/Full-Report.ashx>.

Brown, H.K., V. Cobigo, Y. Lunsky and S.N. Vigod. 2017. "Maternal and Offspring Outcomes in Women with Intellectual and Developmental Disabilities: A Population-Based Study." BJOG 124(5): 757-65.

Brown, H.K., Y. Lunsky, V. Cobigo, A.S. Wilton and S. Vigod. 2016. "Pregnancy in Women with Intellectual and Developmental Disabilities." Journal of Obstetrics and Gynaecology Canada 38(1): 9-16.

Butler, A., C.E. Adair, W. Jones, P. Kurdyak, S. Vigod, M. Smith et al. 2017. Toward Quality Mental Health Services in Canada: A Comparison of Performance Indicators Across 5 Provinces. Vancouver, BC: Centre for Applied Research in Mental Health and Addiction. Retrieved February 26, 2018. <https://www.sfu.ca//dam/sfu/carmha/resources/2017toward-quality-mh/CARMHA\%20REPORT_29\%20Aug_Final.pdf.>

Cobigo, V., H. Ouellette-Kuntz, J.K. Lake, A.S. Wilton and Y. Lunsky. 2013. "Medication Use." In Y. Lunsky, J.E. Klein-Geltink and E.A. Yates, eds. Atlas on the Primary Care of Adults with Developmental Disabilities in Ontario. Toronto, ON: Institute for Clinical Evaluative Sciences and Centre for Addiction and Mental Health. Retrieved February 23, 2018. <http://www.ices.on.ca/ //media/Files/AtlasesReports/2013/Atlas-on-developmental-disabilities/Full-Report.ashx>.

Lin, E., R. Balogh, B. Isaacs, H. Ouellette-Kuntz, A. Selick, A.S. Wilton et al. 2014. "Strengths and Limitations of Health and Disability Support Administrative Databases for Population-Based Health Research in Intellectual and Developmental Disabilities." Journal of Policy and Practice in Intellectual Disabilities 11(4): 235-44.

Lin, E., R. Balogh, C. McGarry, A. Selick, K. Dobranowski, A.S. Wilton and Y. Lunsky. 2016a. "Substance-Related and Addictive Disorders among Adults with Intellectual and Developmental Disabilities (IDD): An Ontario Population Cohort Study.” BMJ Open 6(9): e011638.

Lin, E., R. Balogh, A. Selick, K. Dobranowski, A.S. Wilton and Y. Lunsky. 2016b. Adults with Developmental Disabilities Plus a Mental Illness or Addiction (DD-Plus). Toronto, ON: Health Care Access Research and Developmental Disabilities Program, Centre for Addiction and Mental Health. Retrieved February 26, 2018. <https:// www.porticonetwork.ca/documents/38160/99698/DD+Plus_final + report+\%28August $+17+2016 \% 29$.pdf/c70a84ae-ea20-42c7-abfbc2091e8cc5c8>.
Lunsky, Y., N. Garcin, D. Morin, V. Cobigo and E. Bradley. 2007. "Mental Health Services for Individuals with Intellectual Disabilities in Canada: Findings from a National Survey." Journal of Applied Research in Intellectual Disabilities 20(5): 439-47.

Lunsky, Y., E. Lin, R. Balogh, R. Klein-Geltink, A.S. Wilton and P. Kurdyak. 2012. "Emergency Department Visits and Use of Outpatient Physician Services by Adults with Developmental Disability and Psychiatric Disorder." Canadian Journal of Psychiatry 57(10): 601-07.

Lunsky, Y., R.S. Balogh, V. Cobigo, B.J. Isaacs, E. Lin and H.M.J. Ouellette-Kuntz. 2013a. "Excellent Care for All Adults with Developmental Disabilities: Conclusion." In J. Lunsky, J.E. Klein-Geltink and E.A. Yates, eds. Atlas on the Primary Care of Adults with Developmental Disabilities in Ontario. Toronto, ON: Institute for Clinical Evaluative Sciences and Centre for Addiction and Mental Health. Retrieved February 23, 2018. <http://www.ices. on.ca/ / /media/Files/Atlases-Reports/2013/Atlas-on-developmentaldisabilities/Full-Report.ashx>.

Lunsky, Y., J.E. Klein-Geltink and E.A. Yates, eds. 2013b. Atlas on the Primary Care of Adults with Developmental Disabilities in Ontario. Toronto, ON: Institute for Clinical Evaluative Sciences and Centre for Addiction and Mental Health. Retrieved February 23, 2018. $<$ https://www.ices.on.ca/ / media/Files/Atlases-Reports/2013/Atlason-developmental-disabilities/Full-Report.ashx>.

Lunsky, Y., J.K. Lake, R. Balogh, R. Weiss and S. Morris. 2013c. "A Review of Canadian Mental Health Research on Intellectual and Developmental Disabilities." Journal of Mental Health Research in Intellectual Disabilities 6(2): 106-26.

Lunsky, Y., A. Durbin, H.K. Brown, S. Bansal, M. Heifetz and T. Antonio. 2017. "Health Profiles and Associated Service Use Among Adults with HIV and Intellectual and Developmental Disabilities." AIDS 31(5): 697-705.

Mental Health Commission of Canada. 2013. "Making the Case for Investing in Mental Health in Canada.” Retrieved February 23, 2018. $<$ https://www.mentalhealthcommission.ca/sites/default/files/2016-06/ Investing_in_Mental_Health_FINAL_Version_ENG.pdf>.

Ratnasingham, S., J. Cairney, J. Rehm, H. Manson and P.A. Kurdyak. 2012. Opening Eyes, Opening Minds: The Ontario Burden of Mental Illness and Addictions Report. Toronto, ON: Institute for Clinical Evaluative Sciences and Public Health Ontario. Retrieved February 23, 2018. <https://www.ices.on.ca/ //media/Files/Atlases-Reports/2012/ Opening-eyes-Opening-minds/Full-report.ashx>.

Sullivan, W.F., J.M. Berg, E. Bradley, T. Cheetham, R. Denton, J. Heng et al. 2011. "Primary Care of Adults with Developmental Disabilities: Canadian Consensus Guidelines." Canadian Family Physician 57(5): 541-53.

Weiss, J.A., B. Isaacs, H. Diepstra, A.S. Wilton, H.K. Brown, C. McGarry et al. 2018. "Health Concerns and Health Service Utilization in a Population Cohort of Young Adults with Autism Spectrum Disorder." Journal of Autism and Developmental Disorders 48(1): 36-44. 


\section{About the Authors}

Yona Lunsky, PhD, is the director of the Azrieli Centre for Adult Neurodevelopmental Disabilities and Mental Health, director of the Health Care Access Research and Developmental Disabilities (H-CARDD) Program at the Centre for Addiction and Mental Health and an adjunct scientist at ICES. She can be contacted atYona.Lunsky@camh.ca.

Robert Balogh, $\mathrm{PhD}$, is an assistant professor at the University of Ontario Institute of Technology and an adjunct scientist at ICES. He can be reached at robert.balogh@uoit.ca.

Anna Durbin, $\mathrm{PhD}$, is a scientist at the Centre for Urban Health Solutions at the Li Ka Shing Knowledge Institute of St. Michael's Hospital. She can be contacted at DurbinAn@smh.ca.
Avra Selick, MA, is a research coordinator in the Provincial System Support Program at the Centre for Addiction and Mental Health. She can be contacted at avra.selick@camh.ca.

Tiziana Volpe, PhD, is a research coordinator in the Provincial System Support Program at the Centre for Addiction and Mental Health. She can be contacted at tiziana.volpe@camh.ca.

Elizabeth Lin, $\mathrm{PhD}$, is an independent scientist in the Provincial System Support Program at the Centre for Addiction and Mental Health and an adjunct scientist at ICES. She can be contacted at elizabeth.lin@camh.ca.

\section{Let's talk.}

\section{Longwoods.com}

facebook.com/LongwoodsPublishingCorporation

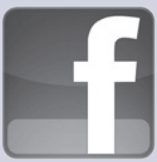

